\title{
Retraction Note: Colorectal Infraperitoneal Anastomosis: The Effects of Perioperative Supplemental Oxygen Administration on the Anastomotic Dehiscence
}

\author{
Mario Schietroma • Francesco Carlei • \\ Emanuela Marina Cecilia • Federica Piccione • \\ Zuleyka Bianchi • Gianfranco Amicucci
}

Published online: 13 November 2013

(C) 2013 The Society for Surgery of the Alimentary Tract

Retraction to: J Gastrointest Surg (2012) Volume 16, Issue 2, pp 427-434

DOI 10.1007/s11605-011-1717-1

The Editors-in-Chief are retracting this article due to similarities to a previously published work entitled "Incidence, consequences, and risk factors for anastomotic dehiscence after colorectal surgery: a prospective monocentric study" by Nicolas C. Buchs, Pascal Gervaz, Michelle Secic, Pascal Bucher, Béatrice Mugnier-Konrad, and Philippe Morel, which was published in the International Journal of Colorectal Disease [(2008) 23; 265-270 DOI: 10.1007/s00384-007-0399-3]. The similarities include text repetition and data and statistics in Dr. Schietroma's Table 5 being similar to Dr. Buchs' Table 3.

The online version of the original article can be found at http://dx.doi.org/ 10.1007/s11605-011-1717-1.

M. Schietroma · F. Carlei • E. M. Cecilia · F. Piccione •

Z. Bianchi $\cdot$ G. Amicucci

Department of Surgery, University of L'Aquila,

Coppito, L'Aquila, Italy

M. Schietroma $(\triangle)$

Università degli Studi di L'Aquila, Facoltà di Medicina e

Chirurgia, Dipartimento di Scienze Chirurgiche,

Presso Ospedale S. Salvatore Edificio Delta 6,

67100 Coppito, L'Aquila, Italy

e-mail: schietroma2001@yahoo.it 